\title{
Derivation of sheep embryonic stem cells under optimized conditions
}

\author{
Marcela Vilarino ${ }^{1}$, Delia Alba Soto ${ }^{1}$, Yanina Soledad Bogliotti ${ }^{1}$, Leqian Yu²,3, \\ Yanli Zhang ${ }^{1}$, Chunsheng Wang ${ }^{1}$, Erika Paulson ${ }^{1}$, Cuiqing Zhong ${ }^{4}$, Miaohan Jin, \\ Juan Carlos Izpisua Belmonte', Jun Wu $\mathrm{u}^{2,3}$ and Pablo Juan Ross $\mathbb{B}^{1}$ \\ ${ }^{1}$ Department of Animal Science, University of California Davis, Davis, California, USA, ${ }^{2}$ Department of Molecular \\ Biology, University of Texas Southwestern Medical Center, Dallas, Texas, USA, ${ }^{3}$ Hamon Center for Regenerative \\ Science and Medicine, University of Texas Southwestern Medical Center, Dallas, Texas, USA and ${ }^{4}$ Gene Expression \\ Laboratory, Salk Institute for Biological Studies, La Jolla, California, USA \\ Correspondence should be addressed to JWu or P J Ross; Email: Jun2.Wu@utsouthwestern.edu or pross@ucdavis.edu
}

\begin{abstract}
Until recently, it has been difficult to derive and maintain stable embryonic stem cells lines from livestock species. Sheep ESCs with characteristics similar to those described for rodents and primates have not been produced. We report the derivation of sheep ESCs under a chemically defined culture system containing fibroblast growth factor 2 (FGF2) and a tankyrase/Wnt inhibitor (IWR1). We also show that several culture conditions used for stabilizing naïve and intermediate pluripotency states in humans and mice were unsuitable to maintain ovine pluripotency in vitro. Sheep ESCs display a smooth dome-shaped colony morphology, and maintain an euploid karyotype and stable expression of pluripotency markers after more than $\mathbf{4 0}$ passages. We further demonstrate that IWR1 and FGF2 are essential for the maintenance of an undifferentiated state in de novo derived sheep ESCs. The derivation of stable pluripotent cell lines from sheep blastocysts represents a step forward toward understanding pluripotency regulation in livestock species and developing novel biomedical and agricultural applications.

Reproduction (2020) $160761-772$
\end{abstract}

\section{Introduction}

Livestock embryonic stem cells (ESCs) have the potential to transform animal biotechnology by improving traits of agricultural significance, generating biomedical models as well as facilitating the study of pluripotency. Due to their unlimited in vitro proliferative capacity, ESCs could be used to introduce complex genetic modifications that enable the generation of animals with desirable agricultural traits as well as modeling human diseases. Recent advancements in targeted genome editing, especially the CRISPR-Cas9 system, have facilitated the introduction of complex genomic alterations, which when combined with somatic cell nuclear transfer (SCNT), represents a powerful platform for transgenic animal production (Gao et al. 2017). Although it remains controversial that success of SCNT is affected by the differentiation state of the donor nucleus (Chung et al. 2014), it was reported that a higher proportion of clones derived from ESC nuclei developed to term when compared to clones derived from somatic cell nuclei, suggesting an advantage of using ESCs as nuclei donors for SCNT (Rideout et al. 2000). ESCs also represent a robust in vitro model for drug discovery, as well as a means for testing the effects of teratogens and toxins on development (Tandon \& Jyoti 2012). Additionally, ESCs have the ability to generate a variety of lineages in vitro, which makes them a good model for studying cellular differentiation (Ezashi et al. 2016). Given the anatomical and physiological similarities that exist between human and livestock species, ESCs are also relevant for expanding their use in regenerative medicine through the creation of animal organ donors (Rashid et al. 2014, Wu et al. 2016).

As a large livestock species, sheep are docile and share similarity with humans in physiology and size, and have been used as models for vaccine development, asthma pathogenesis and treatment, and optimization of drug delivery, among others (Scheerlinck et al. 2008). In addition, sheep have a set of well-established in vitro fertilization procedures.

Until recently, stable ESC lines from livestock species have been difficult to derive and maintain, and ESCs homologous to those described for rodents and primates have not been produced (Ezashi et al. 2012, Malaver-Ortega et al. 2012, Park \& Telugu 2013, Koh \& Piedrahita 2014, Soto \& Ross 2016). Several reports attribute this failure to the lack of appropriate culture 
conditions for derivation and maintenance of ESCs in large animal species (Gandolfi et al. 2012, Soto \& Ross 2016). The number of reports describing livestock ESCs lines increased drastically in the last 10 years (reviewed by Navarro et al. 2019). In sheep, the most successful study was published in 2011 (Zhao et al. 2011), where stable sheep ESCs were derived and cultured for more than 30 passages in $\mathrm{N} 2 \mathrm{~B} 27$ medium supplemented with GSK3 $\beta$ inhibitor (GSK3 $\beta$ i) and FGF2. These cell lines pass the pluripotency assays and formed teratoma, however, teratomas did not have the endoderm component, and they were unable to contribute to chimeras when injected in blastocysts.

In mice, pluripotent stem cells (PSC) have been broadly classified into two pluripotency states, naïve and primed, based on their molecular similarity either to the naïve epiblast in pre/peri-implantation inner cell mass (ICM) or the epiblast from peri-gastrulation embryos (Nichols \& Smith 2009, De Los Angeles et al. 2015). In this regard, mouse ESCs (mESCs) and mouse epiblast stem cells (mEpiSCs) are considered in the naïve and primed pluripotent state, respectively. Although conventional human ESCs are derived from pre-implantation embryos, they resemble mEpiSCs rather than mESCs and are considered to be primed (Tesar et al. 2007). Functionally, naïve mESCs can efficiently contribute to high-grade chimeras when introduced into a blastocyststage embryo. In contrast, although primed mEpiSCs can differentiate into cells from three germ layers in vitro, they do not efficiently generate blastocyst chimeras (Tsukiyama \& Ohinata 2014). Naïve and primed PSCs are cultured in distinct culture conditions: mESCs are conventionally cultured in serum-containing medium supplemented with leukemia inhibitory factor (LIF) and mEpiSCs culture contains fibroblast growth factor 2 (FGF2) and activin A. A ground-state culture condition containing LIF and small molecule inhibitors of ERK1/2 and GSK3 $\beta \mathrm{i}$ ( $2 \mathrm{iL}$ ) has been developed that enables the derivation of ESCs from non-permissive mouse strains as well as rat blastocysts (Buehr et al. 2008, Ying et al. 2008). Human PSCs exhibit overt differentiation in $2 \mathrm{iL}$ culture and does not support the generation of naïve human ESCs and iPSCs (Ying et al. 2008, Hanna et al. 2010, Takashima et al. 2014). In 2013, the first culture medium (NHSM) for the derivation of naïve-like human ESCs from human blastocyst and iPSCs from human fibroblasts was reported (Gafni et al. 2013). Since then, a number of naïve-like culture conditions have been published (Theunissen et al. 2014, Irie et al. 2015, Guo et al. 2016). Furthermore, culture conditions that support the generation of intermediate PSCs with both naïve and primed pluripotency features have also been reported: PSCs cultured in a modified mEpiSC medium containing FGF2, Activin-A and GSK3 $\beta$ i (Factor et al. 2014) showed features characteristic of both mESCs and mEpiSCs (Tsukiyama \& Ohinata 2014, Wu et al. 2017). In addition to naïve cultures, primed PSC cultures have also undergone significant improvement over the past decades. For example, the Essential $8^{\mathrm{TM}}$ Medium (E8) is a xeno-free and feeder-free medium specifically developed for primed hPSCs that has demonstrated the ability to maintain pluripotency in multiple PSC lines (Chen et al. 2011).

Recently, a novel primed PSC culture was developed for both mouse and human. PSCs grown in this condition display region-selective properties (designated as rsPSCs), and unlike conventional mEpiSCs and primed human PSCs, they selectively engraft to the posterior epiblast of gastrula-stage mouse embryos. The human rsPSC culture consists of the custom mTeSR1 base media, FGF2 and IWR1, a tankyrase and WNT pathway inhibitor (CTFR medium) (Wu et al. 2015). Using CTFR medium, we were able to efficiently derive bovine ESCs from blastocysts (CTFR-bESCs) (Bogliotti et al. 2018). CTFR-bESCs displayed stable morphology, karyotype, pluripotency marker expression, epigenetic profiles and were able to form teratoma after injection into immunodeficient mice (Bogliotti et al. 2018). In this study, we tested several reported human naïvelike and primed PSC conditions for the derivation and maintenance of sheep ESCs (sESCs).

\section{Materials and methods}

Unless otherwise specified, all chemicals used are from SIGMA.

\section{Embryo production}

In vitro embryo production was carried out as previously described: ovaries were collected from the slaughterhouse and oocytes were aspirated. Cumulus-oocyte-complexes (COC) were selected and in vitro maturation performed in TCM199 supplemented with 10\% Ovine Estrus Serum (OES), oFSH (50 $\mathrm{ng} / \mathrm{mL}$; National Hormone \& Peptide Program, UCLA, CA), bLH (3 mg/mL; Sioux Biochemical), and cysteamine $(0.1 \mathrm{mM})$, for $24 \mathrm{~h}$ in $5 \% \mathrm{CO}_{2}$ with humidified atmosphere at $38.5^{\circ} \mathrm{C}$ (Vilarino et al. 2017). Ovine estrus serum was collected from ewes synchronized to be in heat at the time of blood draw. In vitro fertilization was performed using fresh semen selected by ascendant migration with a swim-up method using Fertilization Medium (Synthetic oviductal fluid (SOF) supplemented with $2 \%$ OES, $10 \mu \mathrm{g} / \mathrm{mL}$ heparin, and $10 \mu \mathrm{g} / \mathrm{mL}$ hypotaurine). Mature oocytes and sperm adjusted to a concentration of $1 \mathrm{x}$ $10^{6} \mathrm{sperm} / \mathrm{mL}$ were co-incubated for $\sim 14 \mathrm{~h}$ in $5 \% \mathrm{CO}_{2}$ with humidified atmosphere at $38.5^{\circ} \mathrm{C}$. Embryos were cultured in KSOM (Evolve, Zenith Biotech) with $4 \mathrm{mg} / \mathrm{mL}$ of BSA under oil at $38.5^{\circ} \mathrm{C}, 5 \% \mathrm{CO} 2$ and $5 \% \mathrm{O} 2$. Blastocysts were collected on day 7 post-fertilization.

\section{Immunosurgery of blastocysts}

Zona pellucida (ZP)-depleted blastocyst was incubated in KSOM medium with 20\% anti-bovine serum (B8270) for $1 \mathrm{~h}$ at $38.5^{\circ} \mathrm{C}$, followed by five washes with synthetic oviductal 
fluid (SOF)-HEPES media. Embryos were incubated in KSOM supplemented with $20 \%$ guinea pig complement (Innovative Research) for $1 \mathrm{~h}$ at $38.5^{\circ} \mathrm{C}$, and washed five times in SOFHEPES. To isolate the ICM a micro-dispenser was used to gently pipette the ICM and remove the trophoectoderm (TE) cells.

\section{Derivation and culture of sESCs}

In vitro produced blastocysts with a well-developed ICM were collected at day 7 of development. Zona pellucida was removed using $1 \%$ pronase during the first minute and carefully washed up to ten times. Embryos were plated in separate wells of a 24-well plate onto a gamma-irradiated mice embryonic fibroblasts (MEF) feeder layer and cultured in six different media: 2iL (Ying et al. 2008), NHSM (Gafni et al. 2013), 4l (Irie et al. 2015), FAC (Wu et al. 2017), E8 (Chen et al. 2011) and CTFR (Wu et al. 2017). CTFR medium is a custom basal medium similar to mTeSR medium that contains low fatty acid BSA (MP Biomedicals NZ) (Ludwig et al. 2006), and supplemented with $20 \mathrm{ng} / \mathrm{mL}$ of human FGF2 (Peprotech) and $2.5 \mu \mathrm{M}$ IWR1 (Bogliotti et al. 2018). Embryos were cultured at $37^{\circ} \mathrm{C}, 5 \% \mathrm{CO} 2$ and humidified atmosphere. After $48 \mathrm{~h}$, embryos that did not attach were pressed onto the bottom of the culture dish using a 22-gauge sterile needle. The media was changed daily for 7 days, outgrowths were dissociated using TypLE (12563011; Gibco) and passaged to new MEF feeders in the presence of Rho kinase (ROCK) inhibitor Y-27632 $(10 \mu \mathrm{M})$. Media was changed daily between passages, and further passages were performed every 4-5 days at a 1:4 split ratio. Feeder-free culture conditions were performed using vitronectin-coated cell culture plates.

\section{Immunofluorescence}

Immunofluorescence was performed as previously described with some minor modifications (Bogliotti et al. 2018). Sheep ESCs were grown to $70-80 \%$ of confluence in fourwell dishes that had a coverslip and a MEF feeder layer. Cells were fixed using 4\% paraformaldehyde in PBS for 10 min at room temperature and washed three times with PBS. Then, cells were blocked for 30 min with PBST $(0.3 \%$ Triton $\mathrm{X}-100$ in PBS) supplemented with $3 \%$ normal donkey serum (NDS). Cells were incubated in primary antibody at 1:300 dilution in PBST $+1 \%$ NDS for $1 \mathrm{~h}$, washed three times for 10 min using PBST $+1 \%$ NDS, and incubated with fluroescentlabeled secondary antibody at 1:500 dilutions for $1 \mathrm{~h}$. Next, cells were washed three times for 10 min each in PBST, and during the last wash, the nuclei were stained with $0.01 \mu \mathrm{g} / \mu \mathrm{L}$ Hoechst 33342 solution (62249, Gibco). The coverslip with the stained cells was removed from the four-well plate and placed in a microscope slide. Approximately $10 \mu \mathrm{L}$ of Prolong (Life Technologies) was added over the cells, and a coverslip was placed on top. Cells were visualized using a Nikon TE2000-U inverted microscope and a Leica TCS SP8 STED 3X Confocal microscope. The primary antibodies used were antiSOX2 (AN579-5M; BioGenex), anti-OCT3/4 (sc-8628; Santa Cruz Biotechnology), anti-CDX2 (MU392A-UC, BioGenex), anti-GATA6 (sc-9055; Santa Cruz Biotechnology) and antiNANOG (14-5768; eBioscience).

\section{Alkaline phosphatase (AP) staining}

AP staining was performed as previously described: CTFR-sESCs (P36) were grown in a four-well plate to $70-80 \%$ confluence and AP Staining was performed using an AP Staining Kit II (000055, Stemgent) according to the manufacturer's instructions. CTFR-sESCs were briefly washed with PBST $(0.05 \%$ Tween 20 in PBS) and fixed for $4 \mathrm{~min}$ at room temperature using a fixation solution that was provided with the kit. The fixed cells were washed once with PBST and incubated with AP Substrate solution (provided with the kit) in the dark at room temperature for $15 \mathrm{~min}$. Then, AP Substrate Solution was removed and cells were washed twice with PBS. The stained cells were observed using a Nikon TE2000-U inverted microscope and images were captured using RI Viewer Imaging Software (Bogliotti et al. 2018).

\section{Karyotyping}

CTFR-sESCs at passage 22 and 38 were grown to $50-60 \%$ of confluence and arrested in the metaphase stage by incubating them for $1 \mathrm{~h}$ with media containing Demecolcine (200 ng/ $\mu \mathrm{L})$. Cells were harvested using TrypLE, washed with PBS, and resuspended in $15 \mathrm{~mL}$ of $0.075 \mathrm{M} \mathrm{KCL}$ solution pre-warmed to $37^{\circ} \mathrm{C}$ (KCL solution was added drop by drop). After a 10 min incubation at $37^{\circ} \mathrm{C}, 10$ drops of a fixative solution were added (3:1, methanol:acetic acid), and centrifugation was performed at $300 \mathrm{~g}$ for $5 \mathrm{~min}$. The supernatant was discarded and the pellet was resuspended in $5 \mathrm{~mL}$ of the fixative solution, incubated for $15 \mathrm{~min}$ and centrifuged. The fixation procedure was repeated three times and cells were resuspended in 100 $\mu \mathrm{L}$ of the fixative solution and chromosome spreads were prepared by dropping cells suspension over cold slides. After drying for 10-15 min the slides were stained with 3\% Giemsa during a $3 \mathrm{~min}$ period. Slides were visualized using a Nikon TE2000-U inverted microscope at 400× magnification.

\section{Cell cycle analysis by flow cytometry}

Cell cycle analysis was performed as previously described: CTFR-sESCs (P23 and P39) and sheep fibroblasts (P11) (used as control) were grown in a 12 -well plate to $70-80 \%$ confluence and dissociated using TrypLE. After dissociation, $1 \times 10^{6}$ cells were washed twice with PBS supplemented with $2 \%$ FBS and $0.1 \% \mathrm{BSA}$, and centrifuged at $200 \mathrm{~g}$ for $5 \mathrm{~min}$. The supernatant was carefully discarded and $1 \mathrm{~mL}$ of ice-cold $70 \%$ ethanol was added dropwise to resuspend the cell pellet with gentle vortexing. Fixed cells were stored at $-20^{\circ} \mathrm{C}$ until analysis was performed. For flow cytometry analysis, cells were centrifuged at $200 \mathrm{~g}$ for $10 \mathrm{~min}$, washed in PBS supplemented with $2 \%$ FBS and $0.1 \%$ BSA, and then in Stain Buffer (554656; BD Pharmingen). A total of $1 \times 10^{6}$ cells were resuspended in 500 $\mu \mathrm{L}$ of propidium iodide (PI)/RNase Staining Buffer (550825; BD Pharmingen) and filtered through a $5 \mathrm{~mL}$ round-bottom polystyrene tube with a cell strainer snap cap (352235; Falcon). Cells were incubated in PI/RNase staining buffer for $15 \mathrm{~min}$, protected from light, and analyzed within $1 \mathrm{~h}$ on a FACScan flow cytometer (Becton Dickinson) equipped with a $488 \mathrm{~nm}$ excitation laser. Before FACS analysis, cells were 
diluted in PBS to $1 \times 10^{6}$ per $\mathrm{mL}$ in order to achieve a flow rate of 400 events per second. All data were acquired and analyzed using CellQuest Pro Software (Becton Dickinson). Cell doublets were gated out on a FL2-A/FL2-W dot plot, and a total of 10,000 events were collected per sample.

\section{$R N A$ extraction and reverse transcription}

Total RNA was isolated from cells (CTFR-sESCs P15 and P31, and sheep fibroblasts) and tissue (sheep brain and liver) using an RNeasy purification kit (74104; QIAGEN) following the manufacturer's instructions. RNA from 10 blastocysts was extracted using an Arctrurs PicoPure RNA Isolation Kit (1220401, Applied Biosystems) according to the manufacturer's procedures. RNA quantity and quality was analyzed using the NanoDrop 2000C Spectrophotometer (ThermoScientific). cDNA synthesis from cells, tissue and embryos was performed using Superscript II Reverse Transcriptase (18064-014, Invitrogen). Quantitative real-time PCR was performed in QuantStudio 3 (Applied Biosystems) in $20 \mu \mathrm{L}$ reactions containing $0.5 \mu \mathrm{L}$ of forward and $0.5 \mu \mathrm{L}$ of reverse primer $(10$ $\mu \mathrm{M}$ stock of each primer), $2 \mu \mathrm{L}$ of the cDNA sample, $7 \mu \mathrm{L}$ of water and $10 \mu \mathrm{L}$ of Fast SYBR Green Master Mix (00575141, Applied Biosystems). Each sample was run in duplicate for each gene and the sequence of the gene-specific primer sets are provided in the Supplementary Table 1 (see section on supplementary materials given at the end of this article). Relative transcript abundance was calculated using the comparative CT method $(\triangle \mathrm{CT})$ with normalization to GAPDH.

\section{RNA sequencing}

The ICM and TE of two in vitro produced day 7 sheep embryos were mechanically cut using a micro-blade attached to the micromanipulators of an inverted microscope. The TE was washed in PBS, collected in a tube, snap-frozen in liquid nitrogen and stored at $-80^{\circ} \mathrm{C}$ until library preparation. Immunosurgery was performed on the ICM, then snap-frozen and stored at $-80^{\circ} \mathrm{C}$ as previously described. Libraries from sheep ICM and TE were prepared using an Ovation ${ }^{\circledR}$ SoLo RNA-Seq Library Preparation Kit (NuGEN) and sequenced on an Illumina NextSeq instrument producing 75 pb single-end reads. RNA extraction of sESCs (line A: p28, p46; line B: p13, p24) and fibroblasts was performed using an RNeasy Mini Kit (QIAGEN) using DNase treatment (QIAGEN). RNA was analyzed using a 2100 Bioanalyzer (Aglient Technologies) and libraries were prepared using TruSeq ${ }^{\circledR}$ Stranded mRNA Sample Preparation kit (Illumina) and sequenced on a HiSeq 4000 system (Illumina).

All read sets were trimmed (cutadapt/1.16), and individually mapped to the ovine genome Oar_v4.0 (star/2.6.0c). Htseq (version 0.10.0) was used to generate gene counts and data was normalized as Transcripts per Kilobase Million (TPM). A gene was considered expressed when three or more samples had normalized counts $\geq 10$. DESeq (1.24.0) package was used to determine differential gene expression among fibroblasts and sESCs-LCs (IfCThreshold $=1$, alpha $=0.05$ ) and the package pheatmap (1.0.12) was used for heatmap. Gene Ontology enrichment analysis was performed using the enrichR tool
(Chen et al. 2013) and the Reactome Pathway database (https:// reactome.org/) with $P<0.01$ as a cut-off for significant terms. All bioinformatics analysis was performed in $\mathrm{R}$ 3.6.0.

\section{Teratoma assay}

Immunodeficient NOD mice were injected i.m. with approximately $1 \times 10^{6}$ CTFR-sESCs. The teratoma grew for 14-16 weeks and mice were killed for tissue collection. Teratomas were fixed in $4 \%$ paraformaldehyde and subjected to $\mathrm{H} \& \mathrm{E}$ staining to analyze the lineage phenotype.

\section{Data availability}

The RNA-seq data that support the findings of this study are openly available in NCBI/SRA at https://www.ncbi.nlm.nih. gov/sra, SRA accession: PRJNA609175.

\section{Results}

\section{IWR1 supplementation supports derivation and culture of sESCs}

We tested different culture conditions for the derivation of sESCs. In vitro produced sheep blastocysts were subjected to immunosurgery for ICM isolation, and isolated ICMs plated on MEF under six different culture conditions (Supplementary Fig. 1). ICM outgrowths were passaged once per week until passage three (P3) when colony morphology and pluripotency gene expression were evaluated. Cells cultured in CTFR formed colonies with a round dome-shaped morphology, similar to mESCs. In contrast, cells in other culture conditions appeared differentiated and did not form colonies (Fig. 1A). Immunofluorescence analysis (IF) of P3 cells revealed that only cells cultured in CTFR media expressed pluripotency factors OCT4 and SOX2 (Fig. 1B).

Next, we tested whether CTFR-sESCs can be culture adapted to other conditions that failed to maintain in vivo pluripotent epiblast cells during derivation. P6 CTFR-sESCs were transitioned (50-50\% and then $100 \%$ ) to five other conditions and passaged three times before we performed IF analysis with pluripotency markers (Fig. 2A). IF analysis showed that only the control condition (CTFR) maintained the expression of pluripotency markers SOX2 and OCT4 (Fig. 2B). CTFR-sESCs in other conditions lost dome-shaped morphology after three passages. These findings confirm our derivation results and demonstrate that all tested culture conditions except CTFR medium failed to sustain ovine pluripotency in culture. Because IWR1 is a key component of the CTFR conditions, we tested whether tankryrase inhibition is required for maintenance of pluripotency. We found that withdrawal of IWR1 from CTFR culture resulted in the loss of domed colony morphology after two passages and loss of pluripotency marker expression irrespective of FGF2 concentration (Fig. 3). Additionally, 
A
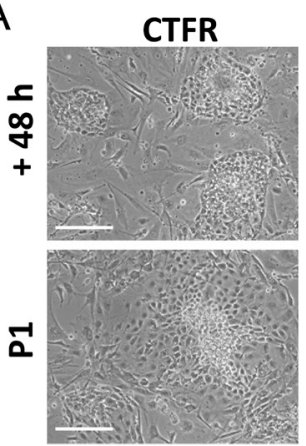

$\stackrel{m}{a}$

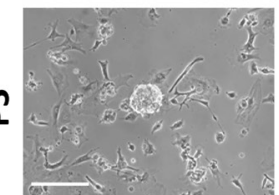

B
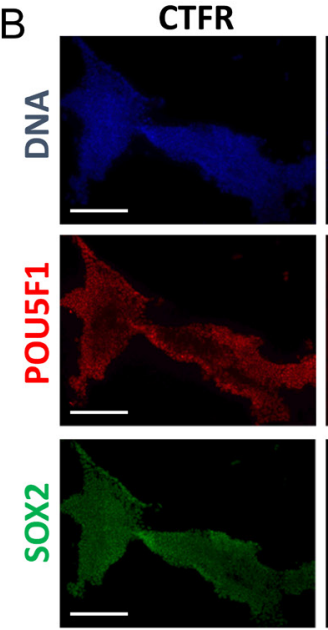

E8
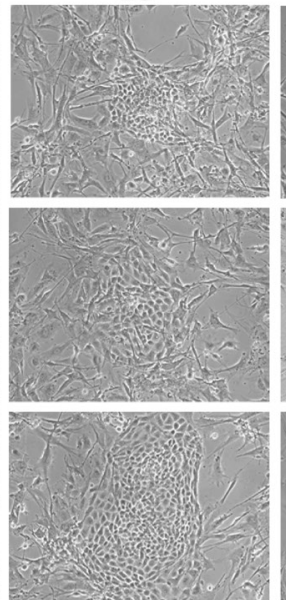

E8
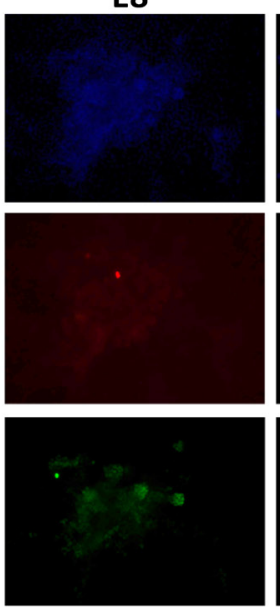

FAC
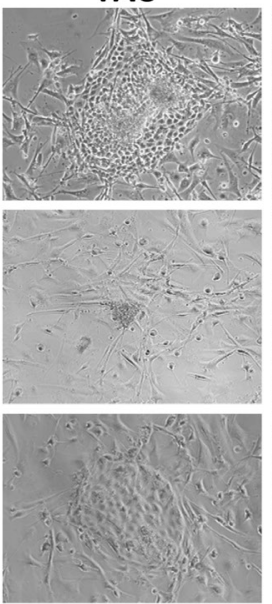

FAC
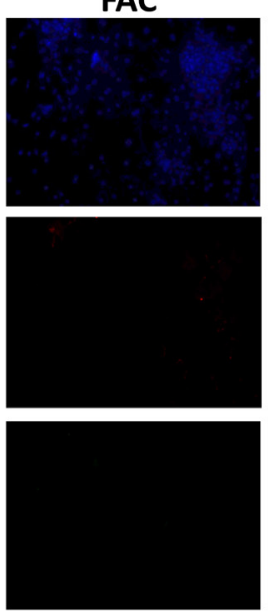

$4 \mid$
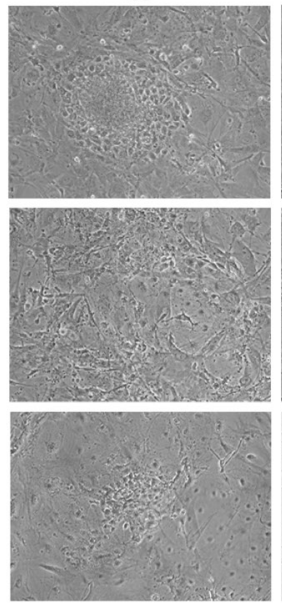

41
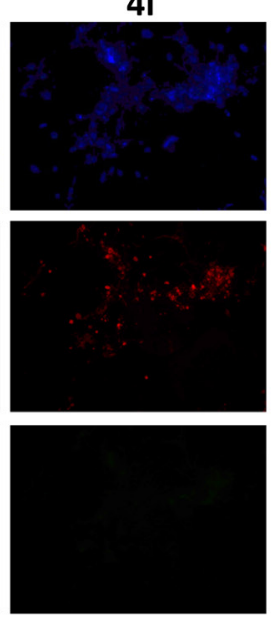

2IL
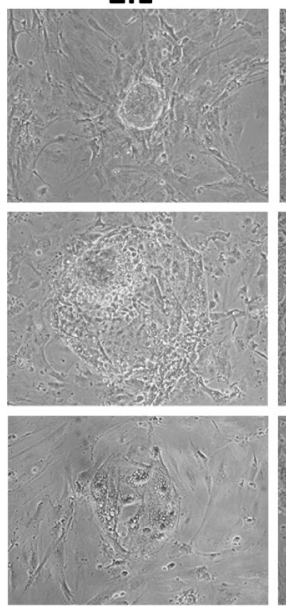

2IL
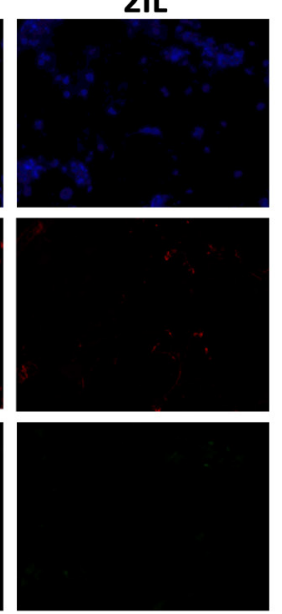

Figure 1 Derivation of sheep ESCs from inner-cell mass (ICM) of sheep embryos under different culture conditions: CTFR (Wu et al. 2017), E8 (Chen et al. 2011), FAC (Wu et al. 2017), 4i (Irie et al. 2015), 2iL (Ying et al. 2008), and NHSM (Gafni et al. 2013). (A) Brightfield images showing colony morphology of sheep ICM culture under six different ESC media conditions at three different time points (48 h after seeding, P1 and P3). (B) Immunofluorescence staining for OCT4 and SOX2 in different ESCs medium conditions at passage three. Scale bars indicate 200 $\mu \mathrm{m}$ (magnification: 10x).

we observed that $4 \mathrm{ng} / \mathrm{mL}$ of FGF2 was insufficient for maintaining normal cell proliferation and pluripotency marker expression (OCT4 and SOX2; Fig. 3).

Collectively, these results show that CTFR condition enabled the derivation of sESCs from sheep ICMs and that IWR1 and high concentrations of FGF2 are required for the maintenance of pluripotency factor expression in sESCs.

\section{CTFR-sESCs exhibit stable morphology and normal karyotype after long term passages}

In addition to isolated ICMs, plating whole sheep blastocysts also resulted in the derivation of sESCs under the CTFR condition (Supplementary Fig. 2). sESCs derived in CTFR condition exhibited a stable domeshaped morphology after long term culture (Fig. 4A and
B). Of note, we observed that CTFR-sESCs colonies were prone to detach when confluent (typically 3-4 days after each passage). Karyotype analysis at passages 21 and 41 indicated that CTFR-sESCs karyotypes were stable with a normal chromosome number of $2 n=54$ (Fig. 4B). Alkaline phosphatase staining showed that CTFRsESCs were positive until at least P36 (Fig. 4B). Analysis of the cell cycle by flow cytometry showed a higher proportion of cells in the $\mathrm{S}$ phase compared to sheep fetal fibroblasts (Fig. 4C), a feature associated with highly proliferative pluripotent cells. Immunofluorescence analysis confirmed the expression of the pluripotency markers OCT4 and SOX2 in long-term cultured CTFRsESCs. Consistent with the epiblast origin of sESCs, the trophoectoderm (TE) and primitive endoderm (PE) markers, CDX2 and GATA6, respectively, were not detected (Fig. 5A). RT-PCR from two late passages of the 


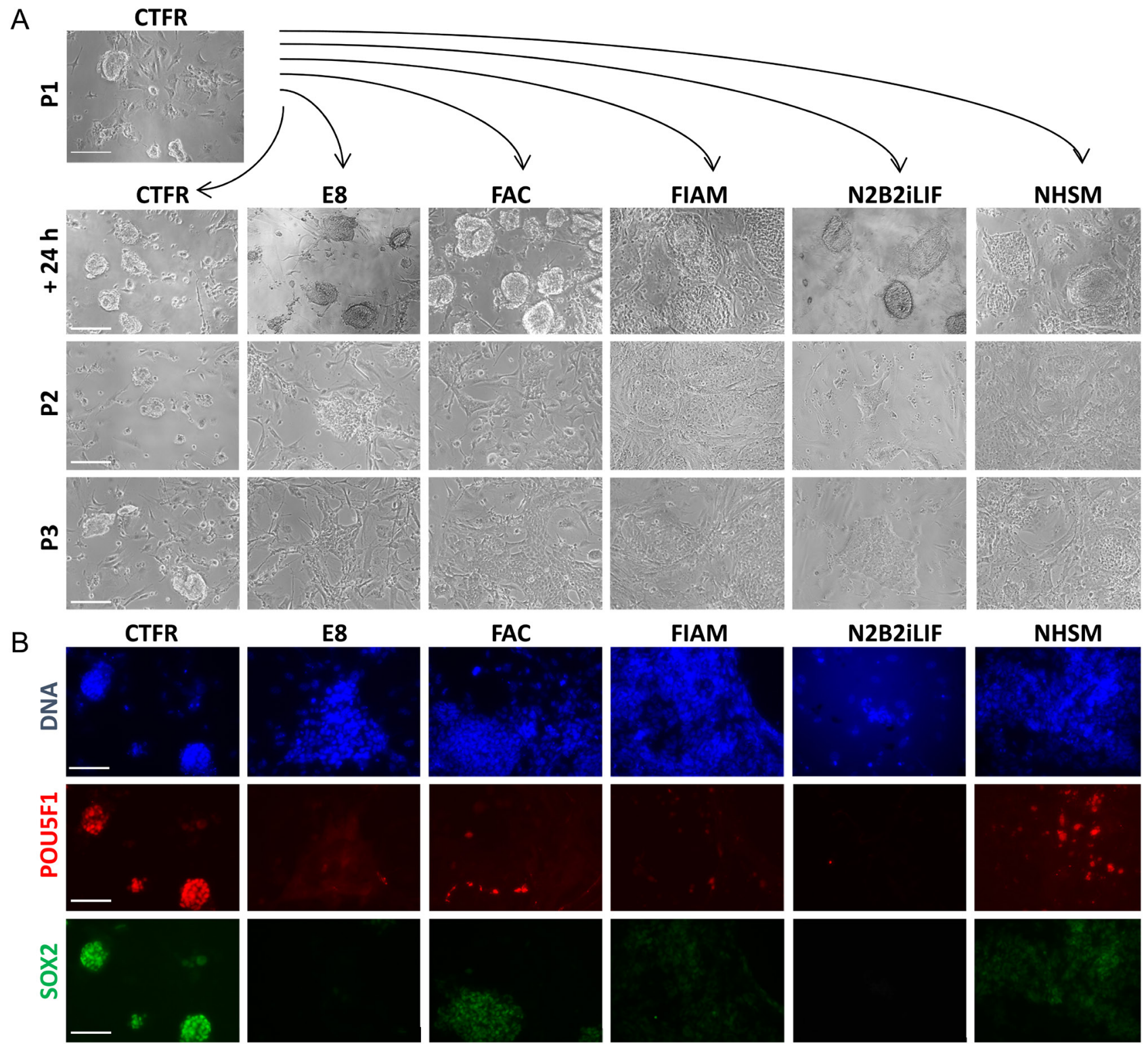

Figure 2 Adaptation of CTFR-sESCs to different culture conditions. (A) Brightfield images showing colony morphology of CTFR-sESCs cultured under six different conditions at three different time points (24 h after media change, P2 and P3). CTFR-sESCs P6 were transitioned (50-50\% and then 100\%) and media was changed daily. (B) Immunofluorescence staining for OCT4 and SOX2 in all the tested ESCs medium conditions at passage three after media change. Scale bars indicate $200 \mu \mathrm{m}$ in A (magnification: 10X) and $100 \mu \mathrm{m}$ in B (magnification: 20x).

CTFR-sESCs confirmed the expression of pluripotency markers OCT4, SOX2 and NANOG, but not the TE marker CDX2, which was expressed in whole blastocysts containing the TE layer. As expected, lineage markers such as PAX6 (ectoderm), FOXA2 (endoderm), and MEOX1 (mesoderm), were not expressed in CTFR-sESCs (Fig. 5B). Moreover, we demonstrated the pluripotency of CTFR-sESC by the formation of a teratoma containing tissues from all three germ layers (Fig. 5C).

Furthermore, we performed RNA-seq using two different CTFR-sESC lines at different passages together with other samples, including ovine fetal fibroblasts, and ICMs and TEs isolated from two sheep blastocysts.
Similar to ICMs, several pluripotency markers were also expressed in CTFR-sESCs, while none of the ICM markers were expressed in fibroblasts (Fig. 6A). Moreover, TE/ PE markers were not expressed in the CTFR-sESCs (Fig. 6A). These results confirm the expression of pluripotency markers and the absence of expression of lineage-specific markers in CTFR-sESCs. Notably, the gene expression profiles of the early and late passages of both CTFRsESC lines clustered closely together, demonstrating the transcriptional stability of CTFR-sESCs over time (Fig. 6B). Still, some variability between cell lines was observed, which could be related to the outbred genetic nature of individual embryos. Additionally, we evaluated 


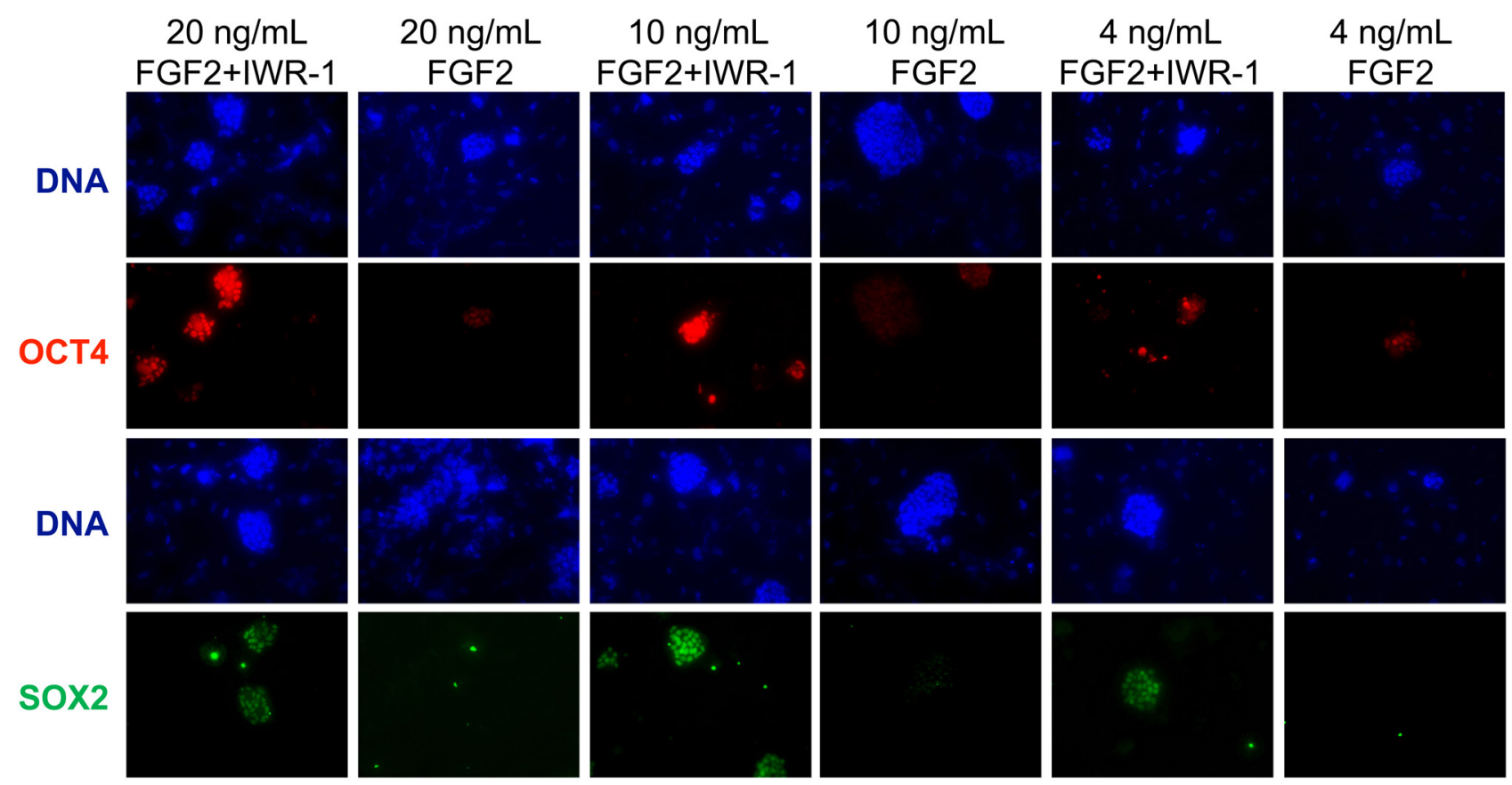

Figure 3 CTFR-sESCs cultured at varying levels of FGF2 with and without IWR1 supplementation. Immunofluorescence staining for OCT4 and SOX2 in the CTFR-sESCs at different FGF concentrations and in the presence or absence of IWR1 (magnification: 10x).

the expression levels of naïve and prime pluripotency markers in CTFR-sESCs. In agreement with bovine CTFR-ESCs (Bogliotti et al. 2018), CTFR-sESC showed primed pluripotency characteristics (Fig. 6C). Among genes differentially expressed between CTFR-sESCs and fibroblast, pluripotency related pathways were the most upregulated gene category in sESCs (Supplementary Fig. 3). Interestingly, NANOG expression was low in one of the cell lines and absent in the other, coinciding with low-level expression detected by RT-PCR. Immunofluorescence analysis confirmed the lack of NANOG expression in CTFR-sESCs (Supplementary Fig. 4).

We also succeeded in culturing CTFR-sESC in a feeder-free condition in which plates were coated with vitronectin and Activin-A (20 ng/mL) was added to the CTFR medium (Tomizawa et al. 2013). After four passages in the feeder-free condition, CTFR-sESCs maintained their typical dome-shaped morphology and pluripotency markers expression (Supplementary Fig. 5).

\section{Discussion}

Stable ESC lines from livestock species have been difficult to derive and maintain, and sheep ESCs homologous to those described for rodents and primates have not been reported (reviewed by Gandolfi et al. 2012). Here, we tested multiple culture conditions for their ability to maintain ovine pluripotency in culture. Our results show that among all the culture conditions tested, only CTFR medium could efficiently derive sESC lines from either plating isolated ICMs or whole sheep blastocysts on MEF feeders. These cell lines maintain a stable morphology, karyotype, and gene expression for more than 40 passages, indicating their potential for self-renewal, and were capable of forming teratomas containing derivatives of the three germ lineages, demonstrating their pluripotency.

Except for CTFR, other naïve, intermediate and primed PSC culture conditions tested, in this study, did not support the maintenance of ovine pluripotency in vitro. Of note, all tested naïve and intermediate media included a GSK3 $\beta$ i (CHIR99021) which can activate the $\mathrm{Wnt} / \beta$-catenin signaling pathway (FAC, $4 \mathrm{i}, 2 \mathrm{iL}$ and NHSM). CTFR culture contains IWR1, a tankyrase inhibitor that stabilizes axin, which then can act as an antagonist for the $\mathrm{Wnt} / \beta$-catenin signaling pathway (Huang et al. 2009), suggesting that canonical Wnt pathway inhibition is the key for the successful derivation of sESCs. However, it is not clear whether IWR1 enables ESCs derivation because of its effect on tankyrase inhibition, axin stabilization, $\beta$-catenin degradation, or a combination of these effects (reviewed by Navarro et al. 2019). Also, the inability of E8 medium (which contains FGF2 and Nodal) to stabilize ovine pluripotency in culture further support the indispensable role of the IWR1. Accordingly, removing IWR1 from the CTFR conditions at high and low FGF2 concentrations resulted in cell differentiation and loss of pluripotency. Similar to bovine CTFR-ESCs, suppressing tankyrase/Wnt activity appears to be the key mechanism that promotes stabilization of ovine pluripotency in culture (Bogliotti et al. 2018). The $\mathrm{Wnt} / \beta$-catenin signaling pathway plays 
A

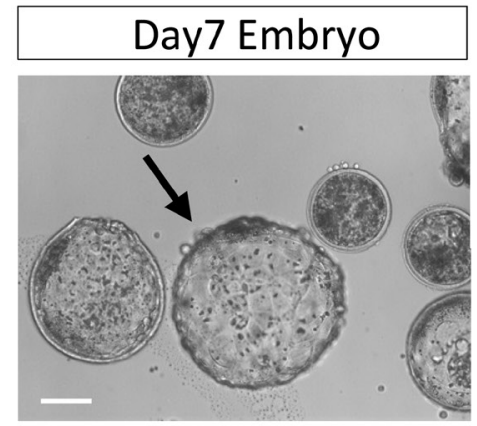

B
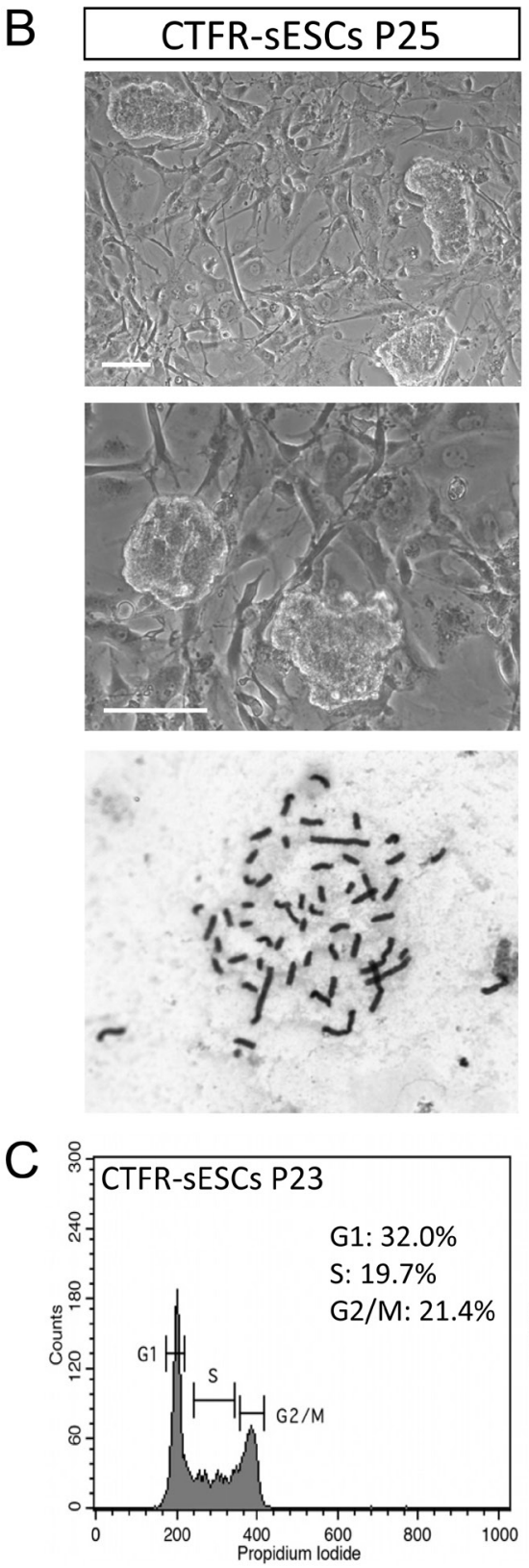
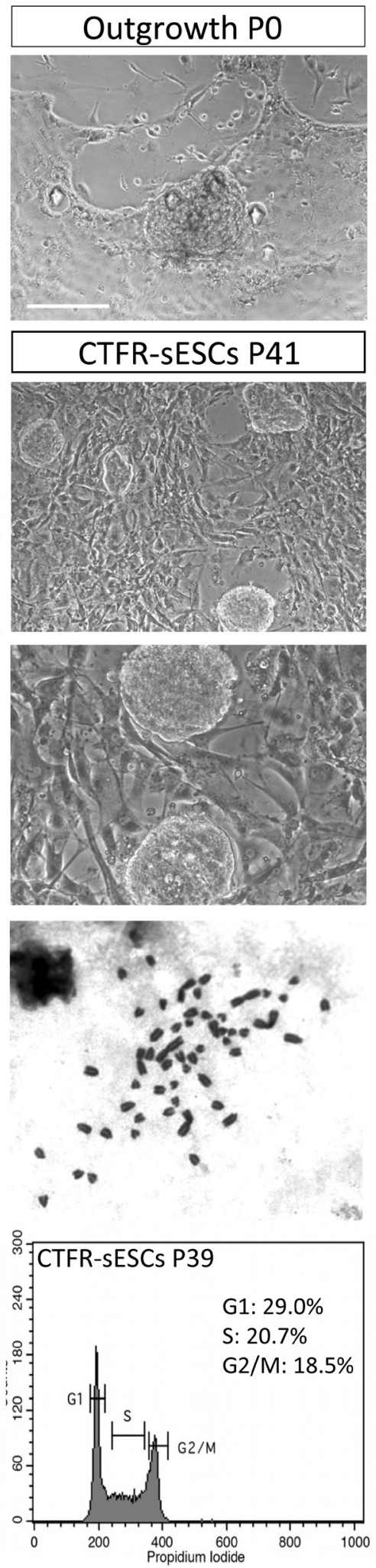
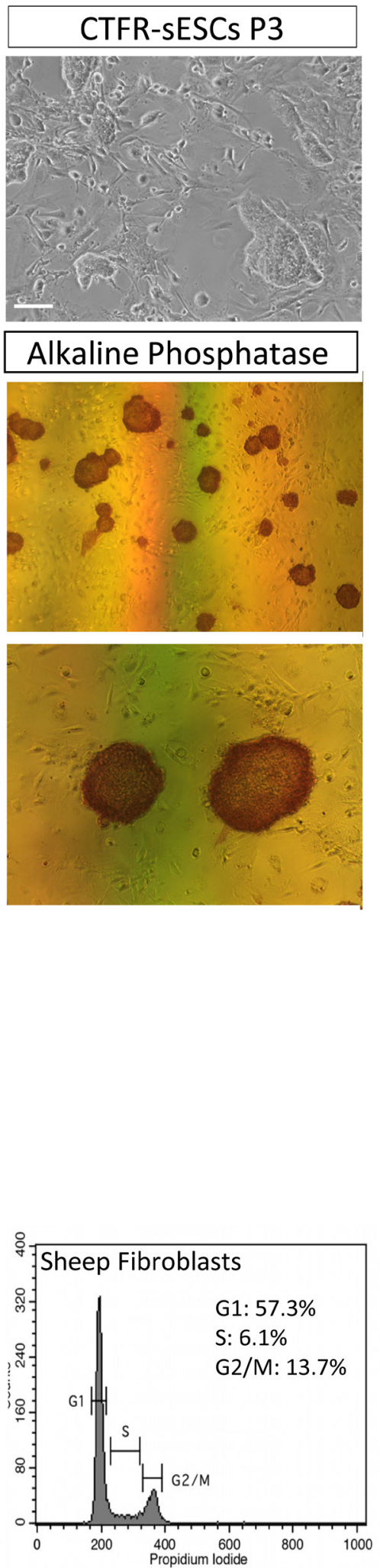

Figure 4 CTFR-sESCs derivation and characterization. (A) Brightfield images showing whole-embryo derivation of CTFR-sESCs under MEF feeders (magnification: 20x objective, for embryo and outgrowth; scale bars $100 \mu \mathrm{m}$ ). (B) Brightfield images (P25 and P41) and alkaline phosphatase (AP) staining (P36) showing CTFR-sESCs colony morphology. Scale bars represent $100 \mu$ m. Karyotype analysis revealed normal chromosome number of $2 n=54$ (magnification: $60 \times$ objective). (C) Cell cycle analysis by flow cytometry of CTFR-sESCs P23 and P39, and sheep fibroblasts. 

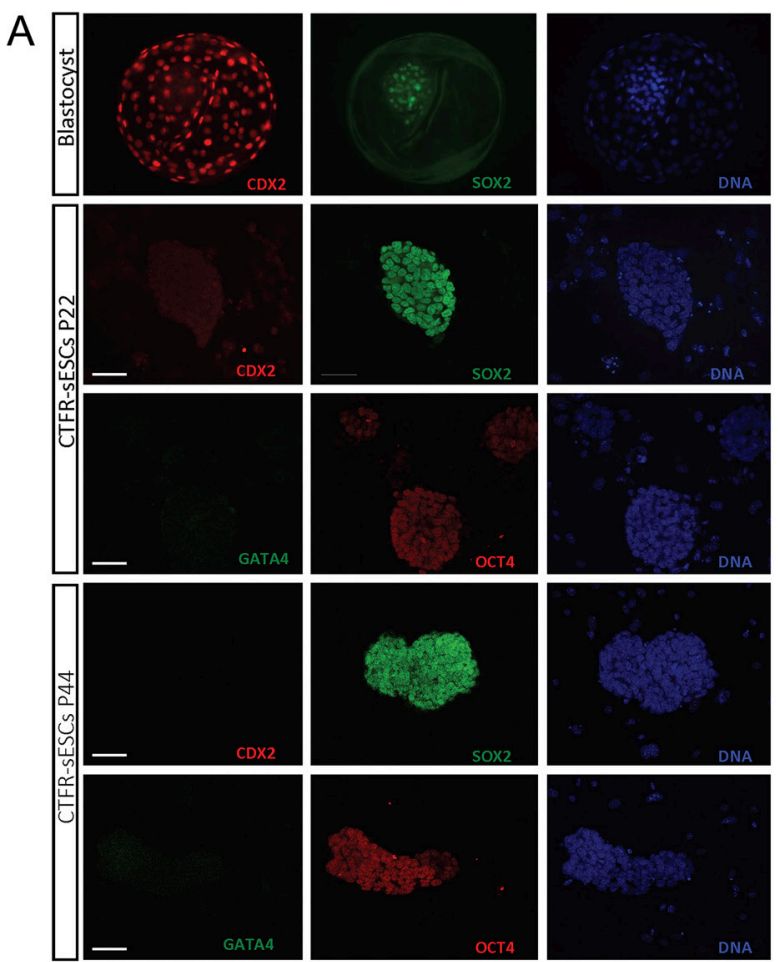

$\left(\frac{10}{2}\right.$
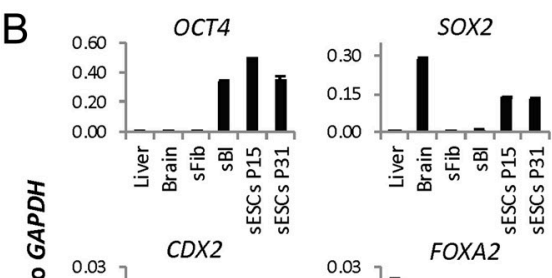

0.00
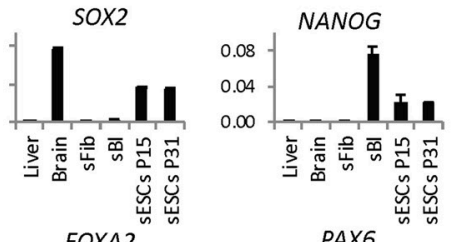

$\left.\begin{array}{ll}\text { \& } & 0.03 \\ \frac{0}{4} & 0.02\end{array}\right]$

홈

0.03
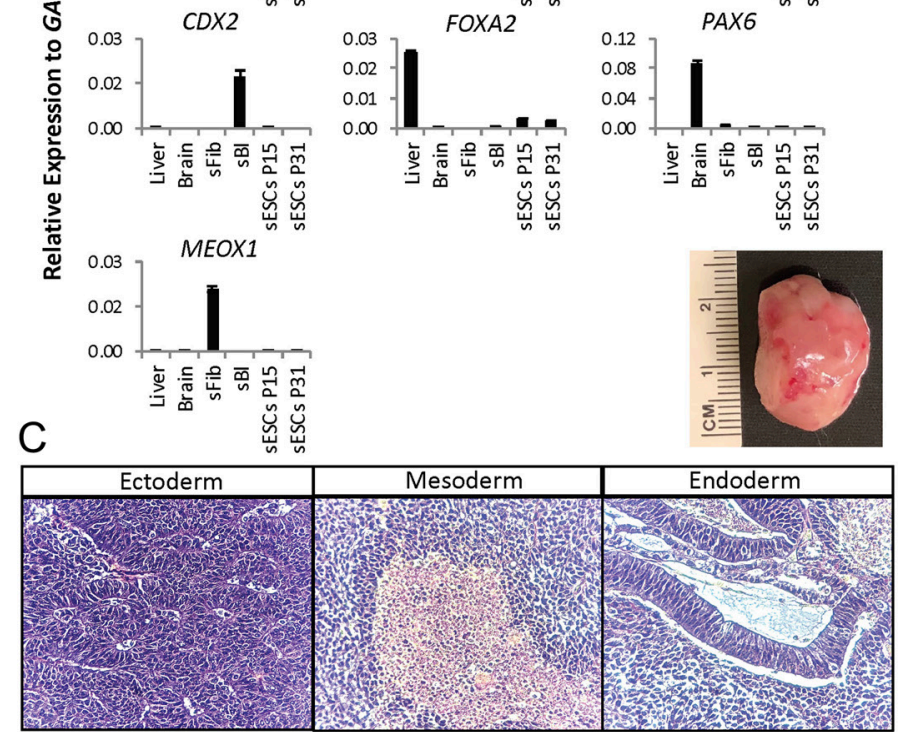

Figure 5 Stable expression of pluripotency markers in CTFR-sESCs. (A) Immunofluorescence staining for OCT4, SOX2, CDX2 and GATA6 in sheep blastocysts (magnification: 20× objective) and CTFR-sESCs P22 and P44 (scale bars, $100 \mu \mathrm{m}$ ). (B) Gene expression analysis using RT-qPCR of ICM pluripotency markers (OCT4, SOX2, NANOG), TE markers (CDX2), endoderm (FOXA2), ectoderm (PAX6) and mesoderm (MEOX1) from two passages of the CTFR-sESCs (P15 and P31), sheep blastocysts, fibroblasts, liver and brain. Relative transcript abundance was calculated using the comparative CT method $(\triangle C T)$ normalized to GAPDH. Error bars represent s.D. of two technical replicates. Representative images of histological sections of H\&E stained teratomas derived from CTFR-sESCs. Tissues of all three germ layers are identified (magnification: $10 \times$ ).

an important role in human and mouse stem cell renewal and differentiation (Sokol 2011) and stimulation of Wnt by GSK3 inhibition is a key component of mouse naïve ESC culture. The $\beta$-catenin destruction complex includes polyposis coli (APC), axin, glycogen synthase kinase (GSK3 $\beta$ ) and casein kinase (CK1a) (Huang et al. 2009). The stability of this complex is regulated by tankyrase 1 and 2 (TNKS1/TNKS2). IWR1 is a small molecule that inhibits TNSK1/2 and stabilizes axin, which blocks the translocation of $\beta$-catenin to the nucleus (Kirubakaran et al. 2014). In addition to the initial reports using IWR1 to stabilize pluripotency in human, mouse and cow ESCs (Wu et al. 2015, Bogliotti et al. 2018), two recent papers reported that IWR1 (or XAV939) is also needed for stabilizing pig pluripotency in vitro (Choi et al. 2019, Gao et al. 2019). These findings, combined with the current work, strongly suggest an important role for tankyrase inhibition, axin stabilization, and/or Wnt inhibition for maintaining pluripotency in a great variety of mammalian species. Further research is required to understand the detailed molecular mechanisms that govern livestock pluripotency.

There are several lines of evidence that might explain the positive effect of $\mathrm{Wnt} / \beta$-catenin inhibition on sESCs pluripotency. A recent study, using bovine embryos showed that endogenous Wnt signaling is not required for blastocysts formation. Embryos were exposed to two different Wnt inhibitors (Wnt-C59 or DKK1) and still were able to reach theblastocyststage. Surprisingly, the presence of Wnt-C59 (non-canonical Wnt pathway inhibitor) increased the number of ICM cells, which suggests that endogenous WNTs regulate ICM proliferation through a signaling pathway independent of $\beta$-catenin (Tribulo et al. 2017). Another study cultured human embryos in the presence of Cardamonin (a $\beta$-catenin inhibitor) and found that transcript levels of pluripotency markers NANOG, OCT4, SOX2 and SALL4 were unaffected, whereas the TE marker CDX2 was downregulated, indicating that $\beta$-catenin degradation or stabilization caused inhibition of TE lineage specification in human blastocysts. Taken together, work in embryos support our results and help explain the positive effect of Wnt inhibition for derivation and maintenance of CTFR-sESCs.

The CTFR-sESCs derived in this study are valuable for addressing basic and translational questions in regenerative medicine (Mahla 2016). Among large animals, the sheep represents an excellent model for cell-based therapeutic approaches because of its similar 


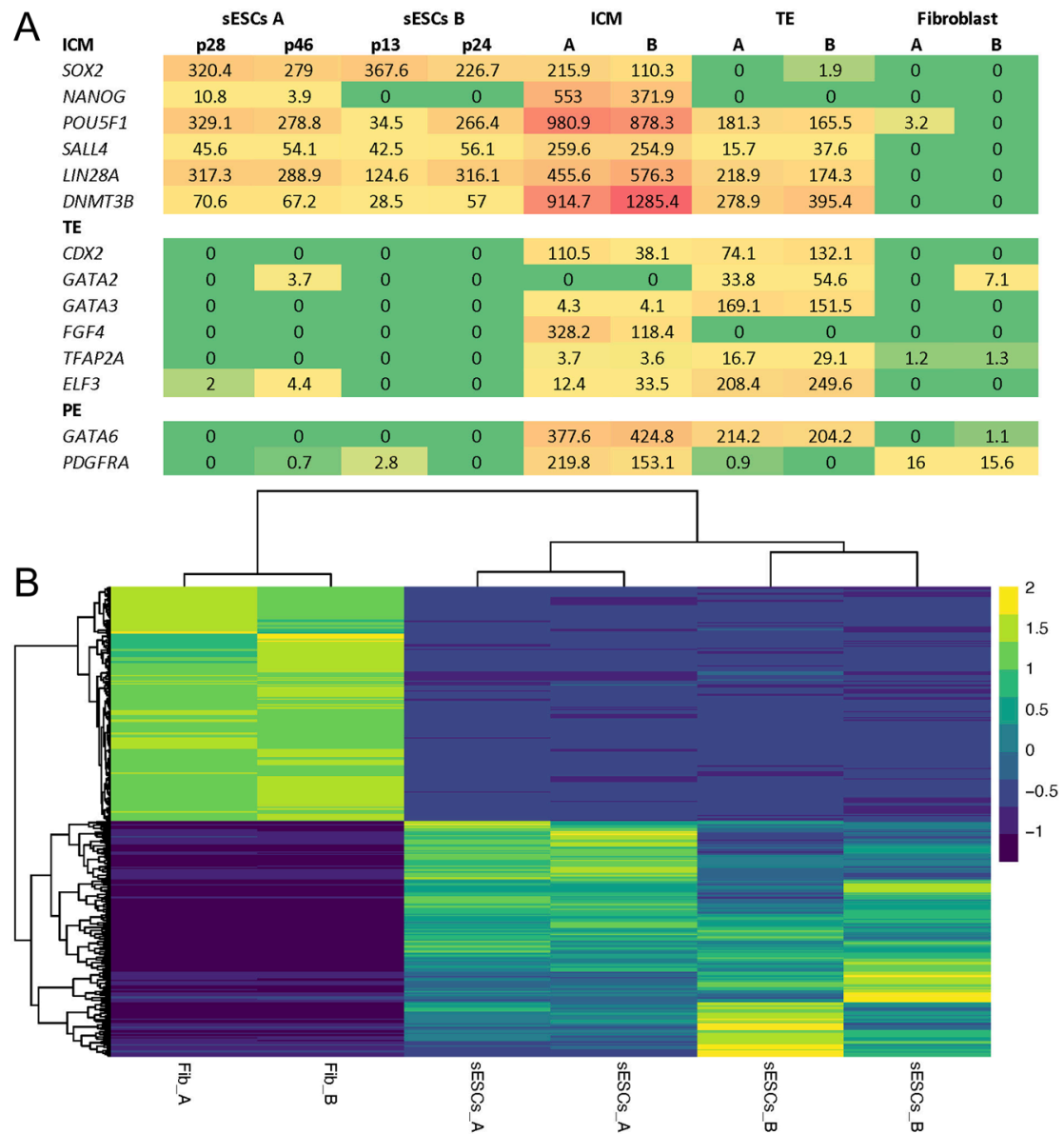

\begin{tabular}{|l|c|c|c|}
\hline & SESCS & ICM & TE \\
\hline TFE3 & 23.6 & 36.0 & 24.9 \\
\hline KLF5 & 10.6 & 1000.7 & 883.6 \\
\hline DUSP14 & 5.7 & 24.0 & 36.6 \\
\hline STAT3 & 5.2 & 76.7 & 2.1 \\
\hline NANOG & 3.7 & 462.5 & 0.0 \\
\hline KLF4 & 3.6 & 298.8 & 22.3 \\
\hline TET2 & 3.0 & 15.2 & 43.4 \\
\hline TEAD4 & 2.2 & 22.8 & 36.1 \\
\hline DNMT3L & 2.1 & 0.0 & 3.2 \\
\hline DUSP5 & 2.0 & 72.2 & 6.7 \\
\hline DUSP10 & 1.8 & 0.0 & 0.4 \\
\hline DUSP3 & 1.3 & 5.3 & 11.3 \\
\hline CD44 & 1.1 & 0.0 & 1.8 \\
\hline ZFP42 & 0.8 & 325.7 & 137.5 \\
TFCP2L1 & 0.6 & 66.5 & 78.4 \\
\hline HORMAD1 & 0.4 & 0.7 & 1.2 \\
\hline TBX3 & 0.3 & 207.1 & 278.3 \\
\hline MAEL & 0.0 & 14.0 & 5.4 \\
\hline DPPA2 & 0.0 & 298.3 & 198.7 \\
\hline SOX6 & 0.0 & 0.5 & 3.1 \\
RFX4 & 0.5 & 0.2 & 0.7 \\
\hline CD47 & 1.7 & 44.1 & 2.1 \\
\hline ZIC1 & 1.8 & 29.3 & 0.0 \\
\hline LMO2 & 1.9 & 30.7 & 0.3 \\
\hline ZNF521 & 2.7 & 0.0 & 0.4 \\
\hline DLL1 & 3.0 & 1.9 & 0.5 \\
\hline MEIS1 & 3.4 & 0.0 & 0.3 \\
\hline HOXB3 & 3.4 & 1.1 & 0.0 \\
\hline TET3 & 3.9 & 6.2 & 27.6 \\
\hline TET1 & 5.3 & 537.3 & 369.6 \\
\hline MEIS2 & 10.4 & 3.9 & 2.0 \\
\hline MYC & 12.4 & 4.0 & 16.5 \\
OTX2 & 16.5 & 28.9 & 4.0 \\
\hline NCAM1 & 19.6 & 1.2 & 0.4 \\
ZIC3 & 30.1 & 71.8 & 0.0 \\
\hline DNMT3A & 30.4 & 265.8 & 289.0 \\
\hline DUSP6 & 55.3 & 67.8 & 47.7 \\
DNMT3B & 55.8 & 1100.1 & 337.2 \\
\hline ZIC2 & 117.2 & 118.7 & 0.7 \\
\hline
\end{tabular}

Figure 6 Transcription profile of CTFR-sESCs. (A) Expression analysis of specific markers for ICM, TE and PE from two independent CTFR-sESCs (A: P28, P46; B: P13, P24), two embryos where ICM and TE were separated and independently sequenced, and two lines of fibroblasts. Transcriptome analysis was performed with RNA-seq (expressed as TPM). In the color scale, red represents highly expressed genes and green represents low/no expression. (B) Heatmap and hierarchical clustering of differentially expressed genes (adjusted $P<0.05$ ) from the two independent CTFR-sESCs (A: P28, P46; B: P13, P24) and the two sheep fibroblast cell lines. (C) Transcriptome analysis of primed and naïve specific gene markers. Data represent the mean of two passages of two independent CTFR-sESCs $(n=4)$ and two independent ICM and TE.

size compared to humans, short gestational interval and well-developed assisted reproductive technologies. Furthermore, stable CTFR-sESC could be harnessed for complex genetic engineering owing to their genetic stability and unlimited proliferative capacity, which could facilitate large-scale genetic modifications for diverse purposes.

In summary, using serum-free medium supplemented with FGF2 and a tankyrase/Wnt inhibitor (IWR1), we were able to de novo derive sESCs from ovine blastocysts that maintain stable colony morphology, normal karyotype, a pluripotent transcriptome, and the ability to form teratomas in vivo after long-term culture. Additionally, sESC can be cultured in a feeder-free condition while maintaining their characteristics. CTFR-sESCs represent a promising tool for use in agriculture and biotechnology and are likely to contribute novel insights into livestock pluripotency and early development.

\section{Supplementary materials}

This is linked to the online version of the paper at http://dx.doi. org/10.1530/REP-19-0606.

\section{Declaration of interest}

The authors declare that there is no conflict of interest that could be perceived as prejudicing the impartiality of the research reported.

\section{Funding}

Work in the laboratory of J C I B was supported by The Moxie Foundation, G. Harold and Leila Y. Mathers Charitable Foundation and Universidad Católica San Antonio de Murcia (UCAM). J $W$ is a Virginia Murchison Linthicum Scholar in Medical Research and funded by Cancer Prevention \& Research Institute of Texas (CPRIT). Work in P J R laboratory 
was partially supported by project W-4171 from USDA-NIFA. $\mathrm{MV}$ was supported by Fulbright and Austin Eugene Lyons Fellowship.

\section{Author contribution statement}

P J R, J C I B, JW and MV conceived the study. P J R, J W, M V, D A S, Y S B, L Y, C Z, M J, Y Z, C W and E P performed experiments and/or analyzed the data. P J R, J W and M V wrote the manuscript.

\section{Acknowledgements}

The authors would like to thank Daniel E Goszczynski for assistance in bioinformatics analysis and Alma Islas-Trejo for her support in molecular biology.

\section{References}

Bogliotti YS, Wu J, Vilarino M, Okamura D, Soto DA, Zhong C, Sakurai M, Sampaio RV, Suzuki K, Izpisua Belmonte JC et al. 2018 Efficient derivation of stable primed pluripotent embryonic stem cells from bovine blastocysts. PNAS 115 2090-2095. (https://doi.org/10.1073/ pnas.1716161115)

Buehr M, Meek S, Blair K, Yang J, Ure J, Silva J, McLay R, Hall J, Ying QL \& Smith A 2008 Capture of authentic embryonic stem cells from rat blastocysts. Cell 135 1287-1298. (https://doi.org/10.1016/j. cell.2008.12.007)

Chen G, Gulbranson DR, Hou Z, Bolin JM, Ruotti V, Probasco MD, SmugaOtto K, Howden SE, Diol NR, Propson NE et al. 2011 Chemically defined conditions for human iPSC derivation and culture. Nature Methods 8 424-429. (https://doi.org/10.1038/nmeth.1593)

Chen EY, Tan CM, Kou Y, Duan Q, Wang Z, Meirelles GV, Clark NR \& Ma'ayan A 2013 Enrichr: interactive and collaborative HTML5 gene list enrichment analysis tool. BMC Bioinformatics 14 128. (https://doi. org/10.1186/1471-2105-14-128)

Choi KH, Lee DK, Kim SW, Woo SH, Kim DY \& Lee CK 2019 Chemically defined media can maintain pig pluripotency network in vitro. Stem Cell Reports 13 221-234. (https://doi.org/10.1016/j.stemcr.2019.05.028)

Chung YG, Eum JH, Lee JE, Shim SH, Sepilian V, Hong SW, Lee Y, Treff NR, Choi YH, Kimbrel EA et al. 2014 Human somatic cell nuclear transfer using adult cells. Cell Stem Cell 14 777-780. (https://doi.org/10.1016/j. stem.2014.03.015)

De Los Angeles A, Ferrari F, Xi R, Fujiwara $Y$, Benvenisty N, Deng $H$, Hochedlinger K, Jaenisch R,Lee S, Leitch HG et al. 2015 Hallmarks of pluripotency. Nature 525 469-478. (https://doi.org/10.1038/ nature15515)

Ezashi T, Telugu BP \& Roberts RM 2012 Induced pluripotent stem cells from pigs and other ungulate species: an alternative to embryonic stem cells? Reproduction in Domestic Animals 47 (Supplement 4) 92-97. (https://doi.org/10.1111/j.1439-0531.2012.02061.x)

Ezashi T, Yuan Y \& Roberts RM 2016 Pluripotent stem cells from domesticated mammals. Annual Review of Animal Biosciences 4 223-253. (https://doi.org/10.1146/annurev-animal-021815-111202)

Factor DC, Corradin O, Zentner GE, Saiakhova A, Song L, Chenoweth JG, McKay RD, Crawford GE, Scacheri PC \& Tesar PJ 2014 Epigenomic comparison reveals activation of 'seed' enhancers during transition from naive to primed pluripotency. Cell Stem Cell 14 854-863. (https://doi. org/10.1016/j.stem.2014.05.005)

Gafni O, Weinberger L, Mansour AA, Manor YS, Chomsky E, Ben-Yosef D, Kalma Y, Viukov S, Maza I, Zviran A et al. 2013 Derivation of novel human ground state naive pluripotent stem cells. Nature 504 282-286. (https://doi.org/10.1038/nature12745)

Gandolfi F, Pennarossa G, Maffei S \& Brevini T 2012 Why is it so difficult to derive pluripotent stem cells in domestic ungulates? Reproduction in Domestic Animals 47 (Supplement 5) 11-17. (https://doi.org/10.1111/ j.1439-0531.2012.02106.x)
Gao Y, Wu H, Wang Y, Liu X, Chen L, Li Q, Cui C, Liu X, Zhang J \& Zhang Y 2017 Single Cas9 nickase induced generation of NRAMP1 knockin cattle with reduced off-target effects. Genome Biology 18 13. (https:// doi.org/10.1186/s13059-016-1144-4)

Gao X, Nowak-Imialek M, Chen X, Chen D, Herrmann D, Ruan D, Chen ACH, Eckersley-Maslin MA, Ahmad S, Lee YL et al. 2019 Establishment of porcine and human expanded potential stem cells. Nature Cell Biology 21 687-699. (https://doi.org/10.1038/s41556-019-0333-2)

Guo G, von Meyenn F, Santos F, Chen Y, Reik W, Bertone P, Smith A \& Nichols J 2016 Naive pluripotent stem cells derived directly from isolated cells of the human inner cell mass. Stem Cell Reports 6 437-446. (https:// doi.org/10.1016/j.stemcr.2016.02.005)

Hanna J, Cheng AW, Saha K, Kim J, Lengner CJ, Soldner F, Cassady JP, Muffat J, Carey BW, Jaenisch R et al. 2010 Human embryonic stem cells with biological and epigenetic characteristics similar to those of mouse ESCs. PNAS $\mathbf{1 0 7}$ 9222-9227. (https://doi.org/10.1073/ pnas.1004584107)

Huang SM, Mishina YM, Liu S, Cheung A, Stegmeier F, Michaud GA, Charlat O, Wiellette E, Zhang Y, Wiessner S et al. 2009 Tankyrase inhibition stabilizes axin and antagonizes Wnt signalling. Nature $\mathbf{4 6 1}$ 614-620. (https://doi.org/10.1038/nature08356)

Irie N, Weinberger L, Tang WW, Kobayashi T, Viukov S, Manor YS, Dietmann S, Hanna JH \& Surani MA 2015 SOX17 is a critical specifier of human primordial germ cell fate. Cell 160 253-268. (https://doi. org/10.1016/j.cell.2014.12.013)

Kirubakaran P, Kothandan G, Cho SJ \& Muthusamy K 2014 Molecular insights on TNKS1/TNKS2 and inhibitor-IWR1 interactions. Molecular Biosystems 10 281-293. (https://doi.org/10.1039/c3mb70305c)

Koh S \& Piedrahita JA 2014 From 'ES-like' cells to induced pluripotent stem cells: a historical perspective in domestic animals. Theriogenology $\mathbf{8 1}$ 103-111. (https://doi.org/10.1016/j.theriogenology.2013.09.009)

Ludwig TE, Bergendahl V, Levenstein ME, Yu J, Probasco MD \& Thomson JA 2006 Feeder-independent culture of human embryonic stem cells. Nature Methods 3 637-646. (https://doi.org/10.1038/nmeth902)

Mahla RS 2016 Stem cells applications in regenerative medicine and disease therapeutics. International Journal of Cell Biology 20166940283. (https://doi.org/10.1155/2016/6940283)

Malaver-Ortega LF, Sumer H, Liu J \& Verma PJ 2012 The state of the art for pluripotent stem cells derivation in domestic ungulates. Theriogenology 78 1749-1762. (https://doi.org/10.1016/j.theriogenology.2012.03.031)

Navarro M, Soto DA, Pinzon CA, Wu J \& Ross PJ 2019 Livestock pluripotency is finally captured in vitro. Reproduction, Fertility, and Development 32 11-39. (https://doi.org/10.1071/RD19272)

Nichols J \& Smith A 2009 Naive and primed pluripotent states. Cell Stem Cell 4 487-492. (https://doi.org/10.1016/j.stem.2009.05.015)

Park KE \& Telugu BP 2013 Role of stem cells in large animal genetic engineering in the TALENs-CRISPR era. Reproduction, Fertility, and Development 26 65-73. (https://doi.org/10.1071/RD13258)

Rashid T, Kobayashi T \& Nakauchi H 2014 Revisiting the flight of Icarus: making human organs from PSCs with large animal chimeras. Cell Stem Cell 15 406-409. (doi:10.1016/j.stem.2014.09.013)

Rideout WM 3rd, Wakayama T, Wutz A, Eggan K, Jackson-Grusby L, Dausman J, Yanagimachi R \& Jaenisch R 2000 Generation of mice from wild-type and targeted ES cells by nuclear cloning. Nature Genetics $\mathbf{2 4}$ 109-110. (https://doi.org/10.1038/72753)

Scheerlinck JP, Snibson KJ, Bowles VM \& Sutton P 2008 Biomedical applications of sheep models: from asthma to vaccines. Trends in Biotechnology 26 259-266. (https://doi.org/10.1016/j. tibtech.2008.02.002)

Sokol SY 2011 Maintaining embryonic stem cell pluripotency with Wnt signaling. Development 138 4341-4350. (https://doi.org/10.1242/ dev.066209)

Soto DA \& Ross PJ 2016 Pluripotent stem cells and livestock genetic engineering. Transgenic Research 25 289-306. (https://doi.org/10.1007/ s11248-016-9929-5)

Takashima Y, Guo G, Loos R, Nichols J, Ficz G, Krueger F, Oxley D, Santos F, Clarke J, Mansfield W et al. 2014 Resetting transcription factor control circuitry toward ground-state pluripotency in human. Cell 158 1254-1269. (https://doi.org/10.1016/j.cell.2014.08.029)

Tandon S \& Jyoti S 2012 Embryonic stem cells: an alternative approach to developmental toxicity testing. Journal of Pharmacy and Bioallied Sciences 4 96-100. (https://doi.org/10.4103/0975-7406.94808) 
Tesar PJ, Chenoweth JG, Brook FA, Davies TJ, Evans EP, Mack DL, Gardner RL \& McKay RD 2007 New cell lines from mouse epiblast share defining features with human embryonic stem cells. Nature 448 196-199. (https://doi.org/10.1038/nature05972)

Theunissen TW, Powell BE, Wang H, Mitalipova M, Faddah DA, Reddy J, Fan ZP, Maetzel D, Ganz K, Shi L et al. 2014 Systematic identification of culture conditions for induction and maintenance of naive human pluripotency. Cell Stem Cell 15 471-487. (https://doi.org/10.1016/j. stem.2014.07.002)

Tomizawa M, Shinozaki F, Sugiyama T, Yamamoto S, Sueishi M, Yoshida T 2013 Activin A is essential for feeder-free culture of human induced pluripotent stem cells. Journal of Cellular Biochemistry 114 584-588. (https://doi.org/10.1002/jcb.24395)

Tribulo P, Leao BCDS, Lehloenya KC, Mingoti GZ \& Hansen PJ 2017 Consequences of endogenous and exogenous WNT signaling for development of the preimplantation bovine embryo. Biology of Reproduction 96 1129-1141. (https://doi.org/10.1093/biolre/iox048)

TsukiyamaT \& OhinataY 2014 A modified EpiSC culture condition containing a GSK3 inhibitor can support germline-competent pluripotency in mice. PLOS ONE 9 e95329. (https://doi.org/10.1371/journal.pone.0095329)

Vilarino M, Rashid ST, Suchy FP, McNabb BR, van der Meulen T, Fine EJ, Ahsan SD, Mursaliyev N, Sebastiano V, Diab SS et al. 2017 CRISPR/ Cas9 microinjection in oocytes disables pancreas development in sheep. Scientific Reports 7 17472. (https://doi.org/10.1038/s41598-017-17805-0)

Wu J, Okamura D, Li M, Suzuki K, Luo C, Ma L, He Y, Li Z, Benner C, Tamura I et al. 2015 An alternative pluripotent state confers interspecies chimaeric competency. Nature 521 316-321. (https://doi.org/10.1038/ nature14413)

Wu J, Greely HT, Jaenisch R, Nakauchi H, Rossant J \& Belmonte JC 2016 Stem cells and interspecies chimaeras. Nature 540 51-59. (https://doi. org/10.1038/nature20573)

Wu J, Platero-Luengo A, Sakurai M, Sugawara A, Gil MA, Yamauchi T, Suzuki K, Bogliotti YS, Cuello C, Morales Valencia M et al. 2017 Interspecies chimerism with mammalian pluripotent stem cells. Cell $\mathbf{1 6 8}$ 473.e15-486.e15. (https://doi.org/10.1016/j.cell.2016.12.036)

Ying QL, Wray J, Nichols J, Batlle-Morera L, Doble B, Woodgett J, Cohen P \& Smith A 2008 The ground state of embryonic stem cell self-renewal. Nature 453 519-523. (https://doi.org/10.1038/nature06968)

Zhao Y, Lin J, Wang L, Chen B, Zhou C, Chen T, Guo M, He S, Zhang N, Liu C et al. 2011 Derivation and characterization of ovine embryonic stem-like cell lines in semi-defined medium without feeder cells. Journal of Experimental Zoology: Part A, Ecological Genetics and Physiology 315 639-648. (https://doi.org/10.1002/jez.715)

Received 12 December 2019

First decision 24 January 2020

Revised Manuscript received 4 August 2020

Accepted 28 August 2020 